\title{
Fatal Streptokinase-Induced Intracerebral Haemorrhage in Cerebral Amyloid Angiopathy
}

\author{
D.A. Ramsay, J.L. Penswick and D.M. Robertson
}

\begin{abstract}
A fatal intracerebral haemorrhage (ICH) associated with streptokinase (SK) treatment of an acute myocardial infarction is described. Autopsy examination showed a lobar $\mathrm{ICH}$ and severe cerebral amyloid angiopathy (CAA). The close temporal relationship between SK administration and intracranial haemorrhage, the absence of pretreatment risk factors for $\mathrm{ICH}$, and the presence of CAA suggests that these are related phenomena. Accordingly: 1. There may be a synergistic relationship between CAA and intracranial haemorrhage induced by fibrinolytic agents; 2. Thrombolytic agents may induce more frequent than expected intracranial haemorrhage in conditions associated with a high incidence of CAA, notably old age and Alzheimer's disease; 3. A regional defect in haemostasis other than vessel fragility may contribute to the intracranial haemorrhagic predisposition of CAA; 4 . Autopsy examination of cases of $\mathrm{ICH}$ is an essential part of the audit of clinical trials of fibrinolytic agents.
\end{abstract}

RÉSUMÉ: Hémorragie intracérébrale fatale induite par la streptokinase dans l'angiopathie amyloïde cérébrale Les auteurs décrivent un cas d'hémorragie intracérébrale (HIC) fatale associée au traitement par la streptokinase (SK) d'un infarctus aigu du myocarde. À l'autopsie, on a constaté une HIC lobaire et une angiopathie amyloïde cérébrale sévère (AAC). La relation temporale étroite entre l'administration de SK et l'hémorragie intracrânienne, l'absence de facteurs de risque pour l'HIC en prétraitement et la présence d'AAC suggèrent que ces phénomènes sont reliés. Par conséquent: I. Il peut y avoir une relation synergique entre l'AAC et l'hémorragie intracrânienne induite par les agents fibrinolytiques; 2 . Les agents thrombolytiques peuvent induire plus fréquemment que prévu une hémorragie intracrânienne dans les affections associées à une incidence élevée d'AAC, particulièrement chez les vieillards et les patients atteints de la maladie d'Alzheimer; 3 . Un défaut régional de l'hémostase autre que la fragilité vasculaire peut contribuer à la prédisposition à l'hémorragie intracrânienne de l'AAC; 4. L'autopsie des cas d'HIC est un élément essentiel de la révision des essais cliniques avec des agents fibrinolytiques.

Fibrinolytic agents have been used sporadically to treat thrombo-occlusive vascular disease for over two decades. ${ }^{1-7}$ The efficacy of streptokinase and related drugs in the management of acute coronary artery thrombosis has been established in several recent clinical trials. ${ }^{8-12}$ Consequently, the use of these drugs and, correspondingly, the incidence of their unwanted effects, are certain to increase over the next few years. Of these side-effects, intracerebral haemorrhage (ICH) is the most devastating and generally augers death or severe morbidity in approximately $0.1 \%$ to $0.7 \%$ of those exposed to the drug. ${ }^{11}$ This complication, which usually develops within the first few hours of treatment, cannot be predicted from routine tests of blood coagulability, and it occurs in the absence of known contraindications to fibrinolytic therapy, including hypertension, a bleeding diathesis, intracranial tumours and a past history of stroke or transient ischaemic attacks. ${ }^{13,14}$ The exclusion of individuals with cere- brovascular disease recalls early attempts to treat these disorders with streptokinase and other thrombolytic agents, which led to unacceptably high rates of intracerebral haemorrhage, related only partly to bleeding into recently infarcted tissue. $2,6.7 .15$

Autopsy studies of streptokinase-induced ICH are sparse, 16 and there appear to be no published neuropathological descriptions of $\mathrm{ICH}$ from individuals who were free of risk factors for intracranial haemorrhage prior to exposure to fibrinolytic agents. This report describes a fatal case of ICH in a previously healthy man who was treated for an acute myocardial infarction with streptokinase. Autopsy examination revealed moderate to severe cerebral amyloid angiopathy (CAA; syn. Congophilic angiopathy, cerebrovascular amyloidosis), which is a major, clinically unrecognisable predisposition to intracranial haemorrhage. 17-23 The conjunction of CAA, fibrinolytic treatment and ICH is of consequence for two reasons: first, in euglycaemic

From the Department of Pathology, Queen's University and Kingston General Hospital, Kingston

Reprint requests to: Dr. D.M. Robertson, Department of Pathology, Richardson Laboratory, Queen's University, Kingston, Ontario, Canada K7L 3N6 
normotensive individuals, non-arteriosclerotic arteriopathies may underlie some, or even all, fatal fibrinolysis-related ICHs autopsy examination of such cases is, accordingly, crucial if the pathophysiology and means of avoidance of such catastrophes are to be defined; second, these observations provide further insight to the pathogenesis of haemorrhage secondary to cerebrovascular amyloidosis.

\section{Material ANd Methods}

\section{Clinical history}

The patient was a 56 year old man who presented to his local hospital on 30th June, 1988, with a 24 hour history of exertional angina pectoris that culminated in a one hour period of crushing chest pain. On admission, he was haemodynamically stable (blood pressure $115 / 80 \mathrm{~mm} \mathrm{Hg}$, pulse $100 / \mathrm{m}$ ) and examination of the cardiovascular and central nervous systems was unremarkable. Subsequent investigation revealed raised serum CK and AST activity (2825 and $262 \mathrm{IU} / \mathrm{L}$ respectively) and an ECG pattern of inferior myocardial infarction. Since there were no contraindications to fibrinolytic therapy, an intravenous infusion of 1.5 megaunits of streptokinase ("Streptase", Hoechst) was given over 1 hour. Eight hours after the start of treatment, he became unresponsive and was transferred to Kingston General Hospital for neurosurgical assessment.

On arrival, he was in coma and showed bilateral extensor posturing in response to painful stimuli. The pupils were unresponsive, the left was fully dilated and the right was at midposition - flame haemorrhages were evident in the left fundus. The CT head scan (Figure 1) showed a large left subdural haemorrhage with left to right transfalceal shift, multifocal left intracerebral temporoparietal haemorrhages and severe cerebral oedema. The coagulation studies were as follows; platelets, $292 \times 10^{9} / \mathrm{L}$; prothrombin time, $25 \mathrm{~s}$ (control 12s); partial thromboplastin time, 79s (control 30s); 2 unit thrombin clotting time (TCT), $>110 \mathrm{~s}$ (normal <26s); 10 unit TCT, $>110$ s (normal $<10 \mathrm{~s}$ ). After correction of

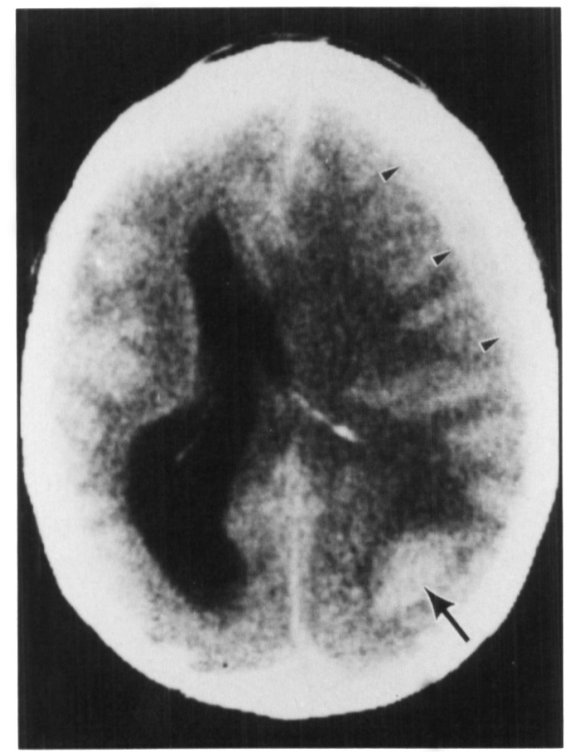

Figure I - This unenhanced pre-operative head CT scan shows, on the left, an occipital intracerebral haemorrhage (arrow) and a large frontoparietal subdural haematoma (arrowheads). Secondary changes include severe left hemispheric cerebral oedema with effacement of the left lateral ventricle, a massive left-to-right shift of the third ventricle and other midline structures, and dilatation of the right lateral ventricle. the clotting defects with vitamin $\mathrm{K}$, fresh frozen plasma and $\varepsilon$-aminocaproic acid, surgical evacuation of the subdural haematoma was performed on $1 / 7 / 88$. Two hours after the completion of surgery the coagulation parameters were normal; serum fibrinogen levels were $1.5 \mathrm{~g} / \mathrm{L}$ and $4.7 \mathrm{~g} / \mathrm{L} 2 \mathrm{~h}$ and $24 \mathrm{~h}$ respectively after surgery (normal 1.7-3.3).

Although he did not regain consciousness, the patient occasionally triggered the ventilator and spontaneously moved all limbs after recovery from anaesthesia; at this stage sluggish symmetrical pupillary light responses were also observed. The day following surgery, focal rightsided seizures emerged - a second CT scan revealed intraventricular haemorrhage and a left temporoparietal blood/fluid level, consistent with an ICH secondary to fibrinolytic therapy; ${ }^{24}$ severe cerebral oedema and transfalceal herniation persisted (Figure 2). An abrupt deterioration occurred on $5 / 7 / 88$, characterised by failure of all routinely tested brain stem reflexes, and the patient died several hours after withdrawal of ventilatory support, within $51 / 2$ days of streptokinase administration.

\section{Pathological Examination}

\section{General autopsy (A-11783)}

The heart weighed $347 \mathrm{~g}$. A $5 \mathrm{~d}$ to $6 \mathrm{~d}$ old haemorrhagic transmural infarct affected $25 \%$ to $30 \%$ of the posterior and septal walls of the left ventricle. The coronary circulation, which was right dominant, showed multifocal triple vessel atherosclerosis with significant $(>80 \%)$ stenosis of the left main, anterior descending and circumflex arteries; old and recent intraplaque haemorrhages were noted at several sites. A rim of mural thrombus was present in the distal right coronary artery, suggesting the site of origin of a recently dislodged thrombus; atheromatous débris were noted in the small intraparenchymal arteries and arterioles adjacent to the infarcted area. Congo red staining of routine sections from the kidneys, liver, heart and lungs showed no evidence of amyloid deposition.

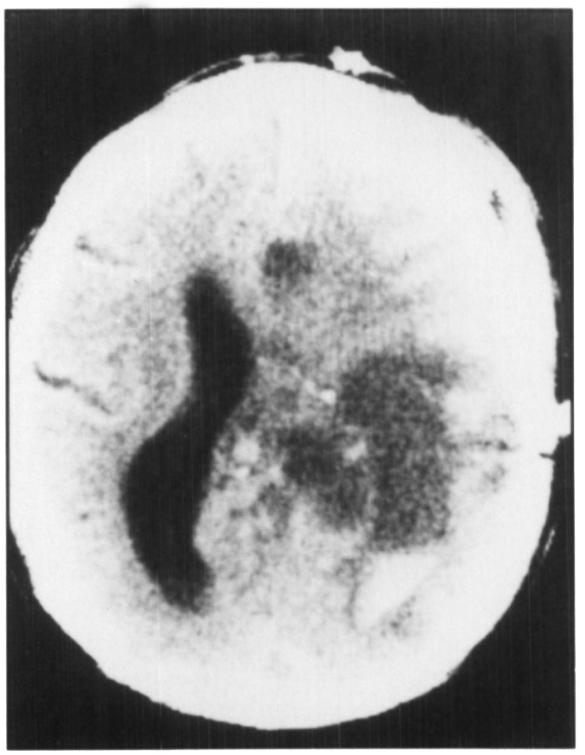

Figure 2 - This unenhanced postoperative head CT scan was done ld after surgical removal of the subdural haematoma ( $f$. Figure (). In contrast to the earlier scan. there is now' a clearly defined left occipital bloodffluid level. Although interpretable as intraventricular, the presence, at this site, of a cavity in the brain slice illustrated in Figure 3 strongly suggests that the blood is intraparenchymal. 


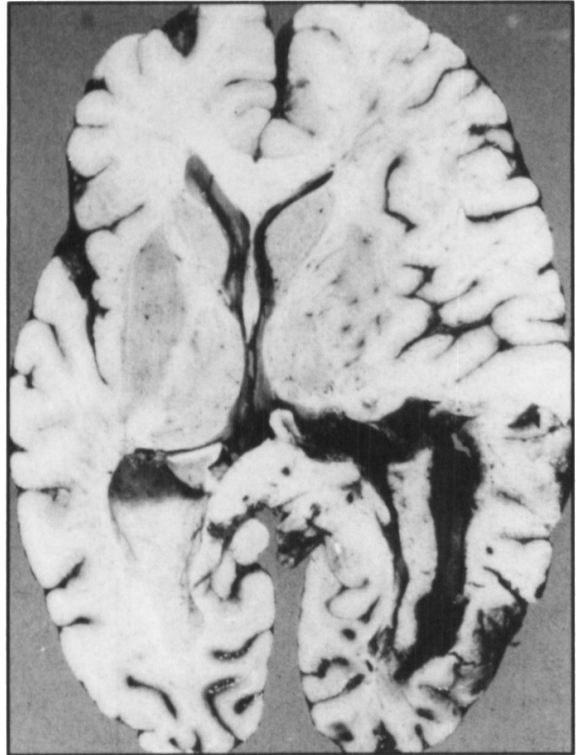

Figure 3 - Extensive tissue destruction and haemorrhage in the left occipital and parietal white matter is shown in this horizontal brain slice. A linear haemorrhagic cavity lies outside, and parallels the curve of, the occipital horn of the left lateral ventricle. Note that the latter communicates with the cavity, which, in higher slices, was in continuity with the subarachnoid space.

\section{Neuropathological examination}

The brain weighed 1562g (NR 1350-1390) and the subarachnoid space was diffusely blood-stained. The intracranial arteries were free of atherosclerosis. There was severe cerebral oedema with marked uncal and left to right transfalceal herniation. A left hemispheric laceration extended from immediately anterior to the occipital notch to the parieto-occipital sulcus. The brain slices showed extensive tissue destruction of, and intraparenchymal haemorrhage into the left occipital, dorsal parietal and caudal temporal regions of the left hemisphere (Figure 3). The laceration that was noted on inspection of the brain surface extended obliquely through the hemisphere to the posterior horn of the left lateral ventricle. The mesencephalon was grossly distorted and Duret haemorrhages were obvious in the mid-brain and pons.

Blocks were taken bilaterally from the frontal (anterior pole), parietal (superior parietal lobule), temporal (middle and inferior temporal gyri) and occipital lobes (occipital pole, including the calcerine cortex), and the hippocampus; tissue was also sampled from the margins of the haemorrhage, basal ganglia (at the level of the mammillary bodies), superior cerebellar lobe (including the dentate nucleus), mid-pons and the medulla oblongata. The material was embedded in paraffin and stained with hematoxylin/eosin/Solochrome, Bodian's silver method, Congo red, thioflavin T, Verhoeff-Van Geison's method for elastin, and Perl's method for iron.

Microscopic examination of the region of haemorrhage and the left occipital cortex showed ischaemic change, including perineuronal vacuolation, neuronal nuclear pyknosis and cytoplasmic hypereosinophilia, scanty polymorphonuclear infiltra- tion and an early gemistocytic astrocytosis. Similar changes were noted in the right calcerine cortex and a small recent microscopic infarct was present in the left frontal lobe.

The typical features of cerebral amyloid angiopathy were widespread in the wall of the small and medium-sized meningeal and cortical arteries, and in the cortical capillaries (Figure 4); these changes were characterised by deposition in the vessel walls of streaks of homogeneously eosinophilic material, which frequently strayed into the adjacent paranchyma to form perivascular plaques (Figure 4). The demonstration at these sites of redgreen birefringence in Congo red-stained sections confirmed the presence of amyloid (Figure 4b), which was most strikingly visualised by fluorescent microscopic examination of thioflavin T-treated sections (Figure 4d). Although the cerebrovascular amyloid deposits were most marked in the occipital regions, they were also prominent in all cerebral cortical regions; modest deposits of amyloid were apparent in the cerebellar meninges, molecular layer and deep white matter - the hippocampus, basal ganglia and brainstem were spared. Secondary degenerative changes in the amyloid-laden vessels were restricted to narrowing of the vessel lumen; other changes, including "double barreling", obliterative change, inflammatory infiltrates, aneurysm formation and fibrinoid necrosis were not apparent $(c f .17,21)$. The grade of amyloid deposition corresponds to Vinter and Gilbert's 25 "severe" (ie, more than 6 cerebral vessel profiles were involved per section), or to "moderate to severe" using Mandybur's classification 21 (ie, CAA change was found in meningeal and cortical vessels, including capillaries, over most of the cortex). Occasional perivascular iron deposition was noted in the occipital regions.

Neuritic plaques were present throughout the frontal, parietal, occipital and temporal cortices, and in the hippocampus (at most, $4-6 / \times 10$ field). There were no neurofibrillary tangles, and neither granulovacuolar degeneration nor Hirano bodies were demonstrable.

\section{Discussion}

The incidence of intracranial haemorrhage related to thrombolytic therapy is low. Review of the literature between 1969 and 1982 showed a $0.7 \%$ incidence of nonfatal and fatal $\mathrm{ICH}$ during fibrinolytic treatment of pulmonary embolism/deep venous thrombosis, peripheral vascular occlusive disease or myocardial infarction. 26 The incidence of $\mathrm{ICH}$ was less $(i e .<0.1 \%)$ in more recent trials, presumably because specific contraindications to fibrinolytic treatment were observed. ${ }^{8.9}$ Consequently, neuropathological accounts of strokes related to the use of streptokinase and related agents are scanty, and those that are available describe haemorrhages associated with significant risk factors, including cerebral infarction ${ }^{15}$ and hypertension. ${ }^{16}$ The present case is an example of a patient in whom no antemortem contraindications to streptokinase use were evident.

Although the series of events in this patient suggest the conventional causal association between ICH and a coagulopathy, with extension of the haemorrhage to the subarachnoid and subdural spaces, the demonstration of CAA indicates that this clinically occult predisposition to intracranial haemorrhage was unmasked by the use of a fibrinolytic agent, which recalls brief comments in the literature regarding the likelihood that acetylsalicylic acid and anticoagulants might precipitate bleeding 
from amyloid-laden vessels. 17,18,22 Accordingly, the lobar location of the ICH with subarachnoid extension is typical of haemorrhage related to cerebrovascular amyloidosis 17.19 .20 .23 and reflects the accumulation of amyloid in the meningeal and cortical vessels. 20,23,25,27 The present case, therefore, raises the possibility that arterial disease, other than atherosclerosis, may underlie many, or all, of the unexpected ICHs occurring in the carefully screened subjects exposed to fibrinolytic agents. Indirect
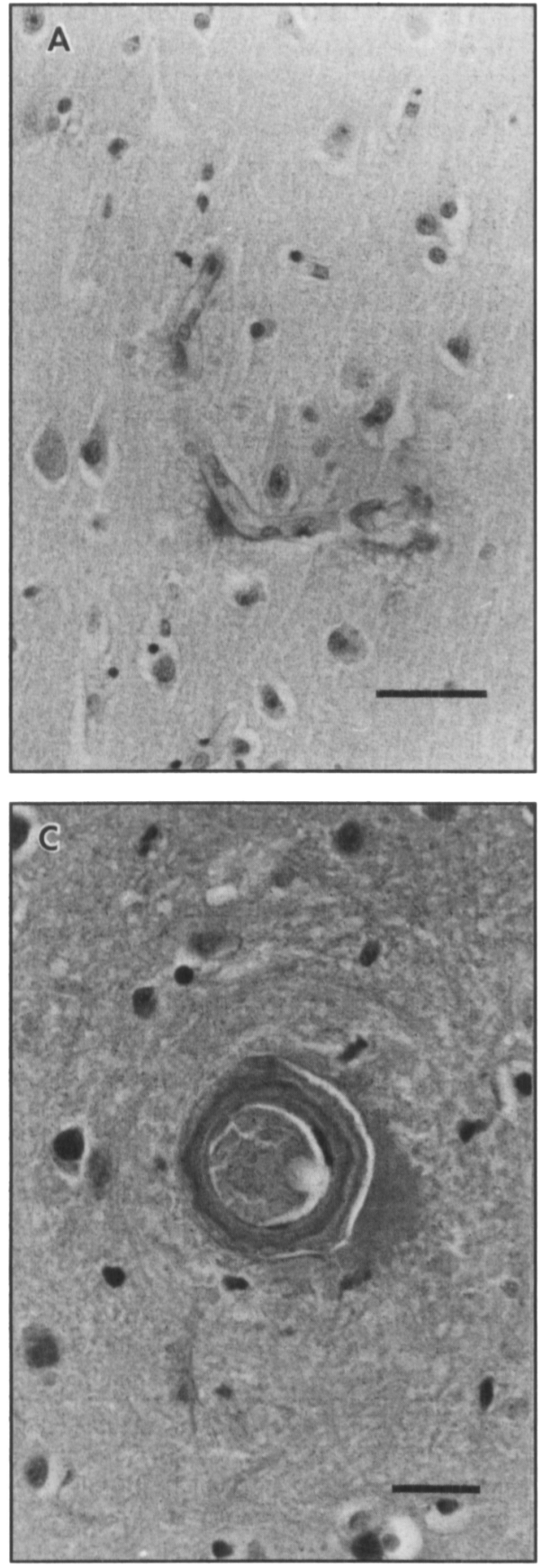

support for this suggestion can be found in reports of trials of tissue plasminogen activator (TPA), a thrombolytic agent that is as effective as streptokinase in the management of myocardial infarction, but with fewer allergic complications 11.12 .28 In these studies, CT head scans of 8 cases with $\mathrm{ICH}$ revealed 5 examples of bleeding in regions that are vulnerable to CAA (cases 2, 4 and 5 in the TIMI II trial; 11 both cases described by Carlson et al.). ${ }^{28}$ Accordingly, thorough neuroradiological and autopsy
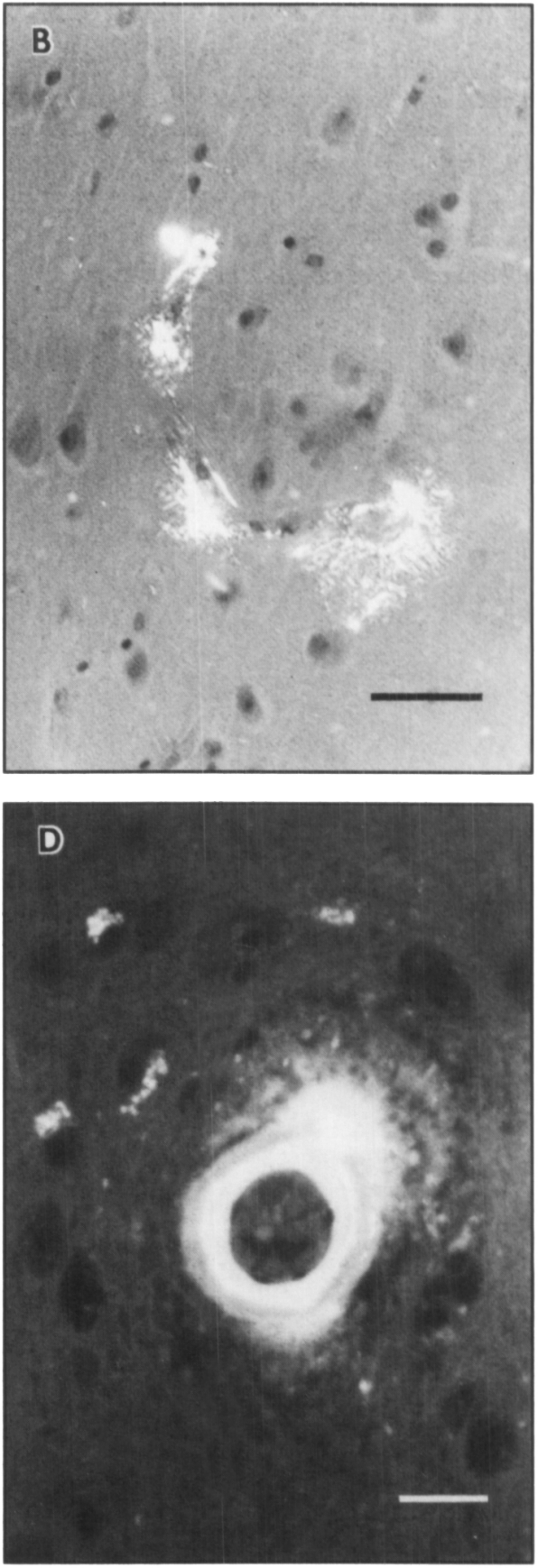

Figure 4 - The typical light microscopic appearances of the vessels in cerebral amyloid angiopathy are demonstrated. A and B $(\times 236$. scale $=50 \mu \mathrm{m}):$ An amyloid-laden capillary stained with Congo red $(A)$ and viewed with polarising filters is shown $(B)$. Note the prominence of the capillary walls, the perivascular amyloid deposits and the bright birefringence of the amyloid. $C(H+E$, Solochrome: $\times 510$, scale $=10 \mu m):$ The walls of this cerebral arteriole, seen in cross-section, are thickened and hyalinised; a perivascular amyloid deposit is obvious. $D$ (thioflavin $T$; $\times$ 480; scale $=10 \mu \mathrm{m})$ : The bright fuorescence of the amyloid-thioflavin $T$ complex clearly illustrates the perivascular amyloid deposit and the extensive infiltration of the cerebral arteriole by the same material. 
examination of the brain should form an important part of the audit of the therapeutic use of thrombolytic agents. Moreover, since the incidence of CAA is high in Alzheimer's disease, ${ }^{29}$ and increases with age from $5 \%$ in the seventh decade to $57 \%$ in the tenth decade, 25 advanced age and dementia should be considered as potential contraindications to fibrinolytic treatment. However, although smaller trials have suggested that the elderly do, indeed, have a greater risk of $\mathrm{ICH}$ during fibrinolytic treatment, ${ }^{30}$ this has not been confirmed in larger recent trials. ${ }^{9}$

The pathophysiology of CAA-associated intracerebral haemorrhages is uncertain. Their occurrence after minor head trauma and neurosurgical procedures implies that amyloid-laden vessels are "brittle" and, thus, are liable to tear as a consequence of trivial mechanical stresses, which perhaps include the normal homeostatic fluctuations in blood pressure; ${ }^{18,31}$ similar suggestions have also been made regarding the haemorrhagic predisposition in systemic amyloidosis. ${ }^{32}$ In addition, the discovery of secondary degenerative cerebrovascular changes, including fibrinoid necrosis and aneurysmal dilatation, in many fatal normotensive cases of $\mathrm{ICH}$ with Congophilic angiopathy has been taken as evidence that aneurysmal rupture leads to hemorrhage, $17,20,21$ by analogy with the proposed vasculopathic aetiology of hypertensive ICH. ${ }^{33}$ However, the observation in the present case that haemorrhage occurred during a period of decreased blood coagulability, in the absence of fibrinoid necrosis and aneurysm formation, suggests that CAA-induced intracerebral haemorrhage may, at least in part, be the result of disordered haemostasis. That this is due to primary deficiencies in circulating coagulation factors is unlikely because the haemorrhagic complications of CAA are restricted to the brain. Consequently, the mechanism of disordered haemostasis is presumably localised to the involved vessels, where several pathogenetic mechanisms could operate. First, leakage of numerous serum proteins into the vessel wall, ${ }^{34}$ and the frequent finding of perivascular haemosiderin deposits, suggests that the endothelium of the Congophilic vessels is, in some way, incompetent. Maintenance of the patency of the vessel lining may, therefore, require greater than usual haemostatic activity, which could readily be overwhelmed by factors such as acetysalicylic acid $^{18,22}$ and, as may apply to the present case, thrombolytic agents at therapeutic serum levels that do not cause haemorrhage from normal blood vessels. Second, by analogy with systemic amyloidosis in which increased fibrinolysis has been demonstrated, ${ }^{35,36}$ the compromised endothelium in CAA may fail to secrete plasmin inhibitors, plasminogen activator inhibitors, or other haemostasis control substances. Third, the capacity for amyloid to bind factor $\mathrm{X}$ and, to a lesser extent, factor IX and prothrombin, ${ }^{37,38}$ may further interfere with local haemostatic activity. Fourth, deposition of amyloid in the subendothelial layer may displace collagen and thus hinder the activity of, or remove an important substrate for, initiating platelet adhesion and the clotting cascade. ${ }^{39}$

In summary, a case of intracranial haemorrhage secondary to streptokinase administration has been described. The discovery of underlying CAA suggests an explanation for some or all of the unexpected intracranial haemorrhages associated with fibrinolytic therapy. The apparent interrelationship between streptokinase, CAA and ICH raises the possibility that local deficiencies in haemostasis, other than, or in addition to, vessel fragility, may explain the haemorrhagic predisposition in cerebrovascular amyloidosis.

\section{ACKNOWLEDGEMENTS}

Our thanks are due to Dr. Sally Ford for assistance with the cardiac pathology, to Barb LeClaire for her skills in the Neuropathology Laboratory, and to Lloyd Kennedy and Blake Gubbins for preparation of the illustrations.

\section{REFERENCES}

1. European working party. Streptokinase in recent myocardial infarction: a controlled multicentre trial. Br Med J 1971; 3: 325 331.

2. Fletcher AP, Alkjaersig N, Lewis M. A pilot study of urokinase therapy in cerebral infarction. Stroke 1976; 7: 135-142.

3. Fletcher AP, Sherry S, Alkjaersig N, et al. The maintenance of a sustained thrombolytic state in man. II. Clinical observations on patients with myocardial infarction and other thromboembolic disorders. J Clin Invest 1959; 38: 1111-1119.

4. Marder VJ, Sherry S. Thrombolytic therapy: current status (second of two parts). New Engl J Med 1988; 318: 1585-1595.

5. Meissner AJ, Misiak A, Ziemski JM, et al. Hazards of thrombolytic therapy in deep vein thrombosis. Br J Surg 1987; 74: 991-993.

6. Meyer JS, Gilroy J, Barnhard M. Therapeutic thrombolysis in cerebral thromboembolism: randomized evaluation of intravenous streptokinase. I $n$ : Millikan $\mathrm{CH}$, Siekert RG, Whisnant JP, eds. Cerebral vascular diseases. New York, Grune and Stratton $1965 ; 200-211$.

7. Meyer JS, Gilroy J, Barnhart MI, et al. Anticoagulants plus streptokinase therapy in progressive stroke. JAMA 1964; 189: 373.

8. Gruppo Italiano per Lo Studio della Streptochinasi nell'Infarcto Miocardico (GISSI). Effectiveness of intravenous thrombolytic treatment in acute myocardial infarction. Lancet 1986; 1:397-401.

9. ISIS-2 (Second International Study of Infarct Survival) Collaborative Group. Randomised trial of intravenous streptokinase, oral aspirin, both, or neither among 17,187 cases of suspected acute myocardial infarction. Lancet 1988; 2: 349-360.

10. Kennedy JW, Martin GV, David KB, et al. The western Washington intravenous streptokinase in acute myocardial infarction randomized trial. Circulation 1988; $77: 345-352$.

11. The Thrombolysis in Myocardial Infarction (TIMI) Phase II Pilot Study. Tissue plasminogen activator followed by percutaneous transluminal coronary angioplasty. J Am Coll Cardiol 1987; 10: 51B-64B.

12. Topol EJ, Morris DC, Smalling RW. A multicenter, randomized, placebo-controlled trial of a new form of intravenous recombinant tissue-type plasminogen activator (Activase) in acute myocardial infarction. J Am Coll Cardiol 1987; 9: 1205-1213.

13. Sherry S. Appraisal of various thrombolytic agents in the treatment of acute myocardial infarction. Am J Med 1987;83 (Suppl 2A): 31-45.

14. Marder VJ, Sherry S. Thrombolytic therapy: current status (first of two parts). New Engl J Med 1988; 318: 1512-1520.

15. Hanaway J, Torack R. Fletcher AP, et al. Intracranial bleeding associated with urokinase therapy for acute ischemic hemispheric stroke. Stroke 1976; 7: 143-146.

16. Gorelick PB, Parikh M, McDonald L. Intracoronary streptokinase and fatal cerebellar hemorrhage. Ill Med J 1987; 171: 28-30.

17. Ozaki H, Reagan TJ, Campbell JR. Clinicopathologic studies of primary cerebral amyloid angiopathy. Mayo Clin Proc 1979; 54: 22-31.

18. Case records of the Massachusetts General Hospital. Case 491982. New Engl J Med 1982; 1507-1514.

19. Gilbert JJ, Vinters HV. Cerebral amyloid angiopathy: incidence and complications in the aging brain. I. Cerebral hemorrhage. Stroke 1983; 14: 915-923. 
20. Kalyan-Raman UP, Kalyan-Raman K. Cerebral amyloid angiopathy causing intracranial hemorrhage. Ann Neurol 1984; 16: 321-329.

21. Mandybur TI. Cerebral amyloid angiopathy: the vascular pathology and complications. J Neuropathol Exp Neurol 1986; 79-90.

22. Case reports of the Massachusetts General Hospital. Case 101988. New Engl J Med 1988; 318: 623-631.

23. Vinters HV. Cerebral amyloid angiopathy: a critical review. Stroke 1987; 18: 311-324.

24. Weisberg LA. Significance of the fluid-blood interface in intracranial hematomas in anticoagulated patients. Comput Radiol 1987; 4: 175-179.

25. Vinters HV, Gilbert JJ. Cerebral amyloid angiopathy: incidence and complications in the aging brain. II. The distribution of amyloid vascular changes. Stroke 1983; 14: 924-928.

26. Aldrich MS, Sherman SA, Greenberg HS. Cerebrovascular complications of streptokinase infusion. JAMA 1985; 253: 1777-1779.

27. Vanley CT, Aguilar MJ, Kleinhenz RJ, et al. Cerebral amyloid angiopathy. Hum Pathol 1981; 12: 609-616.

28. Carlson SE, Aldrich MS, Greenberg HS, et al. Intracerebral hemorrhage complicating intravenous tissue plasminogen activator treatment. Arch Neurol 1988; 45: 1070-1073.

29. Bergeron C, Ranalli RJ, Miceli PN. Amyloid angiopathy in Alzheimer's disease. Can J Neurol Sci 1987; 14: 564-569.

30. Lew AS, Hod H, Cercek B, et al. Mortality and morbidity rates of patients older and younger than 75 years with acute myocardial infarction treated with intravenous streptokinase. Am J Cardiol 1987; $59: 1-5$
31. Torack RM. Congophilic angiopathy complicated by surgery and massive hemorrhage: a light and electron microscopic study. Am J Pathol 1975; 81: 349-366.

32. Yood RA, Skinner M, Rubinow A, et al. Bleeding manifestations in 100 patients with amyloidosis. JAMA 1983; 249: 1322-1324.

33. Cole FM, Yates P. Intracerebral microaneurysms and small cerebrovascular lesions. Brain 1967; 90: 759-767.

34. Powers JM, Schlaepfer WW, Willingham MC, et al. An immunoperoxidase study of senile cerebral amyloidosis with pathogenetic considerations. J Neuropathol Exp Neurol 1981; 40: 592-612.

35. Liebman $\mathrm{H}$, Chinowsky $\mathrm{M}$, Valdin $\mathrm{J}$, et al. Increased fibrinolysis and amyloidosis. Arch Intern Med 1983; 143: 678-682.

36. Takahashi $\mathrm{H}$, Koike $T$, Yoshida $\mathrm{N}$, et al. Excessive fibrinolysis in suspected amyloidosis: demonstration of plasmin- $\alpha_{2}$-plasmin inhibitor complex and von Willebrand factor fragment in plasma. Am J Hematol 1986; 23: 153-166.

37. Furie B, Voo L, McAdam K, et al. Mechanism of factor X deficiency in systemic amyloidosis. New Engl Med J 1981: 304: 827-830.

38. Griepp PR, Kyle RA. Bowie EJW. Factor $X$ deficiency in anyloidosis: a critical review. Am J Hematol 1981; 11: 443-450.

39. Sixma JJ. Role of blood platelets, plasma proteins and the vessel wall in haemostasis. In: Bloom AL, Thomas DP, eds. "Haemostasis and Thrombosis". New York, Churchill Livingstone 1987; 283-311. 